\title{
Live-imaging of Mitochondrial System in Cultured Astrocytes
}

\author{
Jeanne Espourteille ${ }^{1}$, Valentin Zufferey ${ }^{1}$, Jean-Honoré Laurent ${ }^{1}$, Kevin Richetin $^{1}$ \\ ${ }^{1}$ Department of Psychiatry, Center for Psychiatric Neurosciences, Lausanne University Hospital (CHUV) and University of Lausanne
}

\section{Corresponding Author}

Kevin Richetin

kevin.Richetin@chuv.ch

\section{Citation}

Espourteille, J., Zufferey, V., Laurent, J.H., Richetin, K. Live-imaging of Mitochondrial System in Cultured Astrocytes. J. Vis. Exp. (177), e62957, doi:10.3791/62957 (2021).

\section{Date Published}

November 16, 2021

\section{DOI}

$10.3791 / 62957$

URL

jove.com/video/62957

\section{Abstract}

While much attention has been given to mitochondrial alterations at the neuronal level, recent evidence demonstrates that mitochondrial dynamics and function in astrocytes are implicated in cognition. This article describes the method for time-lapse imaging of astrocyte cultures equipped with a mitochondrial biosensor: MitoTimer. MitoTimer is a powerful and unique tool to assess mitochondrial dynamics, mobility, morphology, biogenesis, and redox state. Here, the different procedures for culture, image acquisitions, and subsequent mitochondrial analysis are presented.

\section{Introduction}

Astrocytes are critical players in the maintenance of brain homeostasis. They are perhaps most well known to have significant structural roles in the brain, as part of the bloodbrain barrier ${ }^{1}$ and by supporting neurons and synapses throughout the brain ${ }^{2}$. Astrocyte support of neurons is both structural ${ }^{3}$ and metabolic ${ }^{4,5}$, with astrocytes promoting neurogenesis and synaptogenesis while also providing key metabolites like lactate to active neurons ${ }^{4,6,7}$. Beyond the role of structural support, astrocytes are active cells that take part in $\mathrm{Ca}^{2+}$ signaling and buffering (including spontaneous mitochondrial $\mathrm{Ca}^{2+}$ influxes) ${ }^{8,9}, \mathrm{~K}^{+}$buffering ${ }^{10}$, and can adapt and react to the needs of the brain in times of injury $^{11,12}$. Being such dynamic cells, astrocytes have robust energy requirements, which necessitate an efficient mitochondrial network. These mitochondria also have a crucial role in buffering excessive reactive oxygen species $(\mathrm{ROS})^{13}$. In addition to their individual or local roles of energy generation and ROS buffering, mitochondria function as a network ${ }^{14}$. In this sense, they maintain equilibrium between fissioning and fusioning mitochondria, representing new/reduced mitochondria and older/oxidized mitochondria, respectively ${ }^{15}$. The overall redox state of a cell can be gauged 
by the redox state of the mitochondrial network. In pathology, this is a critical piece of information that can shed light on which cells may not be functioning optimally.

In recent years, many sensors have been developed to study the dynamics and functions of mitochondria in cells. For example, sensors measuring energy exchange (ATP), redox state (NADH/NAD $\left.{ }^{+}, \mathrm{ROS}\right)$, and enzymatic functionality (cAMP, $\mathrm{Ca}^{2+}, \mathrm{Zn}^{2+}$ ) are currently used in the study of mitochondrial function ${ }^{16}$. Among them, MitoTimer permits to follow the changes in mitochondrial morphology (size, shape, surface area), mobility (speed, displacement), and dynamics (fusioning and fissioning events), as well as the overall mitochondrial turnover rate and redox state. MitoTimer is a mutant red fluorescent protein, $\operatorname{drFP} 583^{17}$, with a mitochondrial signal from subunit VIII of human cytochrome c oxidase ${ }^{18,19}$ to visualize newly synthesized mitochondria in green $(488 \mathrm{~nm})$ and oxidized mitochondria in red $(555 \mathrm{~nm})$. Using the green $(488 \mathrm{~nm})$ and red (555 $\mathrm{nm}$ ) fluorescence ratio permits simultaneous evaluation of individual mitochondria, their morphology analysis, fusion/ fission events, and redox state history 20,21 . This unique property can be used to investigate many scientific questions regarding mitochondria's physiological and pathological roles and is therefore very promising for unveiling the underlying mechanisms of mitochondrial dynamics within many different cell types.

We recently developed a new lentiviral vector (LV-G1MitoTimer-MiR124T, hereafter called LV-G1-MitoTimer) to study mitochondria's dynamic and functions specifically in astrocytes in vitro and in vivo ${ }^{22}$. LV-G1-MitoTimer uses a truncated version of the glial fibrillary acidic protein (GFAP) promoter gfaABC1D, with a B3 enhancer (gfaABC1D(B3), hereafter called G1) combined with the previously described
miR124T neuronal detargeting system ${ }^{23}$. It allows exclusive expression of the mitochondrial biosensor in astrocytes in vitro and in vivo ${ }^{22}$. Presented here are the different steps to perform a culture of rat hippocampal astrocytes and equip them with the LV-G1-MitoTimer biosensor, as well as the different microscopy steps to follow the behavior of astrocyte mitochondria during several consecutive hours/days.

\section{Protocol}

The present protocol has been performed with the approval of an ethical committee (agreement VD3602, Lausanne, Switzerland) and follows European guidelines for the use of animals.

\section{Rat hippocampal astrocyte primary culture}

1. Sacrifice five rat pups (Wistar IGS Rat) by decapitation at postnatal day 1-2.

2. Remove the brain and keep it in a Petri dish containing $5 \mathrm{~mL}$ of fresh astrocyte medium (DMEM with GlutaMAX supplemented with $1 \%$ Penicillin/Streptomycin and $10 \%$ fresh Horse Serum).

3. Isolate the hippocampus. Dissociate in $5 \mathrm{~mL}$ of the astrocytic medium by three passages through a $21 \mathrm{G}$ needle and three passages through a $25 \mathrm{G}$ needle.

4. Transfer the dissociated cells to a $15 \mathrm{~mL}$ centrifuge tube and count in a hemocytometer.

5. Plate $20,000-25,000$ cells $/ \mathrm{cm}^{2}$ in multiwell dishes $(9.4$ $\mathrm{mm} \times 10.7 \mathrm{~mm} \times 9.3 \mathrm{~mm}$ ) and store them at $37^{\circ} \mathrm{C}$ under an atmosphere containing $5 \% \mathrm{CO}_{2}$ for the rest of the experiment.

6. Replace the medium entirely at 3 days in vitro (DIV3). 
7. At DIV8, add $0.6 \mathrm{pg}$ of p24 antigen per cell of lentiviral vector coding for mitochondrial biosensor MitoTimer (LVG1-MitoTimer ${ }^{22}$ ) diluted in phosphate-buffered saline $(\mathrm{PBS}+0.01 \% \mathrm{BSA})$.

8. At DIV9, wash with pre-warmed $1 \mathrm{x}$ sterile PBS $\left(37^{\circ} \mathrm{C}\right)$ and add fresh astrocyte medium.

9. At DIV11, perform 2 washes with pre-warmed 1x sterile PBS $\left(37^{\circ} \mathrm{C}\right)$ and add fresh astrocyte medium without phenol red.

NOTE: Choice of the coating depends on the intended assay. As a standard coating for astrocyte primary culture, it is recommended to use $0.2 \mathrm{mg} / \mathrm{mL}$ poly-Dlysine or $8.7 \mu \mathrm{g} / \mathrm{cm}^{2}$ of basement membrane matrix (e.g., Matrigel). To visualize the mitochondrial system of astrocytes, it is essential to work on flattened and stretched cells. In this context, the combination of basement membrane matrix on IBIDI u-slides is the most suitable. It is also crucial not to work with phenol red, which is toxic for the cell under repeated exposure to light.

\section{Long-term monitoring of the mitochondrial system.}

1. Assess the astrocytic mitochondrial system a minimum of 3-5 days after the lentiviral infections with LV-G1MitoTimer (to allow a sufficient level of fluorescence in the mitochondria).

2. Image the cells with an inverted microscope controlled by the acquisition and analysis software and a module for full automation of acquisition.

NOTE: Refer to the Table of Materials for the details of the software and module used in this study.
3. Ensure that the microscope is equipped with a cage incubator (see Table of Materials) to maintain astrocyte culture at $5 \% \mathrm{CO}_{2}$ and $37^{\circ} \mathrm{C}$ throughout the experiment.

4. Capture fluorescence images using sequential excitation at $490 \mathrm{~nm}$ (for green channel) and $550 \mathrm{~nm}$ (for red channel) with detection of green (500-540 nm for green channel) and red (550-600 nm for red channel) fluorescence signals.

5. Select 5 astrocytes per well with a mitochondrial network expressing sufficient levels of LV-G1-MitoTimer using a magnification of $40 x$. Take care to select astrocytes as flat and large as possible and not located in clusters of cells (to work on individual cells).

6. Save the coordinates of the 5 selected cells in an xIm file (map.xIm). This map.xIm allows the user to return to the same cells over time.

7. Acquire image sequences (1 image/s for $60 \mathrm{~s}$ ) using a magnification of $150 x$ (100x oil immersion objective, $1.5 x$ intermediate magnification) for each coordinates.

8. Repeat image acquisition (1 image/s for $60 \mathrm{~s}$ ) for the same astrocytes $6 \mathrm{~h}, 12 \mathrm{~h}$, and $24 \mathrm{~h}$ after treatments.

NOTE: Use a microscope equipped with a fast and efficient autofocus system. In this context, the hardware solution called Perfect Focus System (PFS) was used. PFS uses near-infrared 870-nm LED and CCD line sensors to combat axial focus fluctuations in real-time during long-term imaging investigations.

\section{Analysis of individual mitochondrial morphology and LV-G1-MitoTimer ratio}

NOTE: NIS General Analysis 3 (GA3) from Nikon was used to automatize morphometric analysis in this study. 
1. For each image sequence (baseline, $6 \mathrm{~h}, 12 \mathrm{~h}, 24 \mathrm{~h}$ ), select the first frame for red and green channels by clicking on ND Processing > Select Frame.

2. Merge the red and the green channels by selecting Conversions > Merge Channel.

3. To correct image shading, select Preprocessing > Auto Shading Correction.

4. Apply the Rolling Ball algorithm by selecting Preprocessing > Rolling Ball.

5. To generate binary masks for each mitochondrion, select Segmentation > Threshold.

6. Remove any objects truncated by the border by selecting Binary processing > Touching Border.

7. To measure the surface area, select Measurement > Object Area.

8. To measure the diameter, select Measurement $>\mathbf{E q}$ Diameter.

9. To measure the length, select Measurement > Length.

10. To measure the width, select Measurement $>$ Width.

11. To measure roughness, select Measurement > Roughness.

12. To measure circularity, select Measurement > Circularity.

13. To measure elongation, select Measurement > Elongation.

14. Compose a group (right-click) with the above measurement and rename it as MorphoData.

15. Measure mean green intensity by selecting Measurement > Mean Intensity.
16. Measure mean red intensity by selecting Measurement > Mean Intensity.

17. To measure the Red/Green ratio, select Measurement > Ratio.

18. Compose a group (right-click) with the above measurement and rename it as RatioData.

19. Export the table to a CSV file by selecting Reference > Table to CSV.

20. Save the GA3 script of analysis by selecting Save As NOTE: A non-exhaustive list of the criteria used in this procedure is summarized in Figure 1, Table 1, and Table 2. The analysis GA3 script file is available in supplemental (Supplemental Coding File 1 and Figure 2). The thresholds used were adapted to individualize as many mitochondria as possible (excluding large mitochondrial networks where possible). Wherever possible, mitochondrial networks are either individualized manually or excluded from the analyses. Due to intercellular variability, perform these analyses on at least $20-25$ cells per condition (with a minimum of 50 mitochondria by cell).

\section{Analysis of mitochondrial motility}

NOTE: Due to the high complexity of mitochondrial movements, manual motility analysis is preferred. Here, Nikon's NIS Element system was used to manually track mitochondria.

1. Open the tracking module in NIS, select View $>$ Analysis $>$ Tracking.

2. Click on Define New ROI.

3. With the Automatic Detection Tool, select 25-50 mitochondria on the first image of the image sequence. 


\section{Click on Track Autodetected ROls Analyze.}

5. If necessary, delete the incorrect ROI tracks.

6. Export the table to a CSV file.

NOTE: A non-exhaustive list of the criteria used in this procedure is summarized in Figure 1 and Table 3. A tracking analysis generally gives a path depicting the movements of the center of every tracked object. The different tracking options must be set in the tracking option. Favor the selection of isolated mitochondria that are sufficiently distant from each other to facilitate tracking. Keep only the objects that could consistently be tracked over the whole sequence. Proceed the same way to remove outliers due to bad quality tracking. Consequently, the set of objects analyzed in this section is different from the one analyzed for static morphological analyses and will generally be smaller.

\section{Data transformation, normalization, and statistical analysis}

NOTE: Mainly due to the high heterogeneity of the mitochondria, data generated often have a non-normal distribution.

1. Manually log-transform the measurements prior to processing.

2. For each analysis and time frame, manually normalize the resulting data by the mean of those obtained in the reference acquisition.

3. In addition, consider a second/double normalization to a control condition, for example, to evaluate the effect of a treatment.
4. Then, perform statistical analysis using a two-way matched ANOVA. Here GraphPad Prism V8 was used.

\section{Representative Results}

Primary culture of astrocytes infected with LV-G1MitoTimer exhibited typical mitochondrial networks. Before treatment, astrocytes expressing LV-G1-MitoTimer showed the heterogeneous mitochondrial size and various green/red fluorescence intensities (Figure 3, Figure 4, and Video 1). The mitochondrial system of astrocyte cultures expressing LV-G1-MitoTimer was monitored before and after incubation with $\mathrm{H}_{2} \mathrm{O}_{2}(10 \mu \mathrm{M})$. The different mitochondrial features described above were calculated over $12 \mathrm{~h}$ (every $3 \mathrm{~h}$ ) and normalized (cell by cell) to their initial state. At the morphological level (Figure 3B), the effects of $\mathrm{H}_{2} \mathrm{O}_{2}$ start to be visible at about $6 \mathrm{~h}$. Indeed, the mitochondria were fragmented (decrease of length, surface area, and elongation factor). This fragmentation is even more obvious $12 \mathrm{~h}$ after the treatment. Note that the diameters, widths, and sphericity were not reduced. Concerning redox state and turnover (Figure $3 \mathrm{C}$ ), $3 \mathrm{~h}$ after $\mathrm{H}_{2} \mathrm{O}_{2}$ treatment, the proportion of green mitochondria increased in astrocytes (the consequence of a rapid increase in mitochondrial biogenesis). At $6 \mathrm{~h}$, the green/ red ratio returned to baseline levels, but the number of purely red mitochondria increased significantly from basal levels. After $12 \mathrm{~h}$, the consequences of the oxidative treatment of $\mathrm{H}_{2} \mathrm{O}_{2}$ were visible and resulted in a substantial increase in the ratio and number of red puncta. Concerning the dynamics and mobility (Figure 3D), $3 \mathrm{~h}$ after the treatment, all the criteria were transiently increased. In the longer term (12 h), the mitochondria moved more slowly and over shorter distances. 


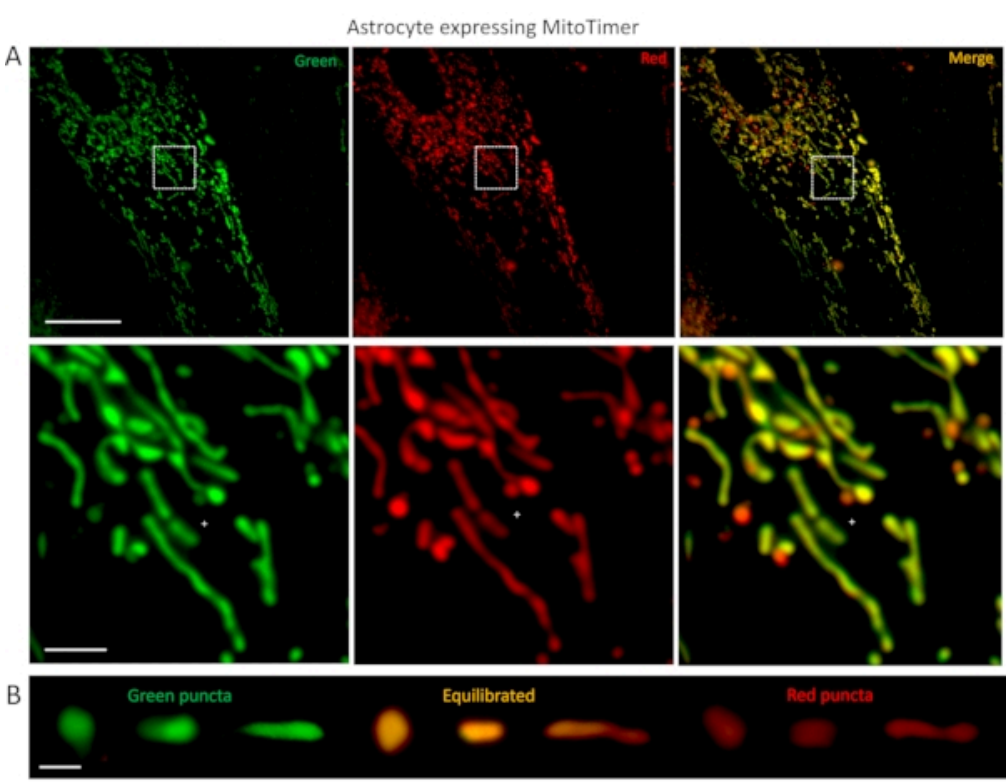

c

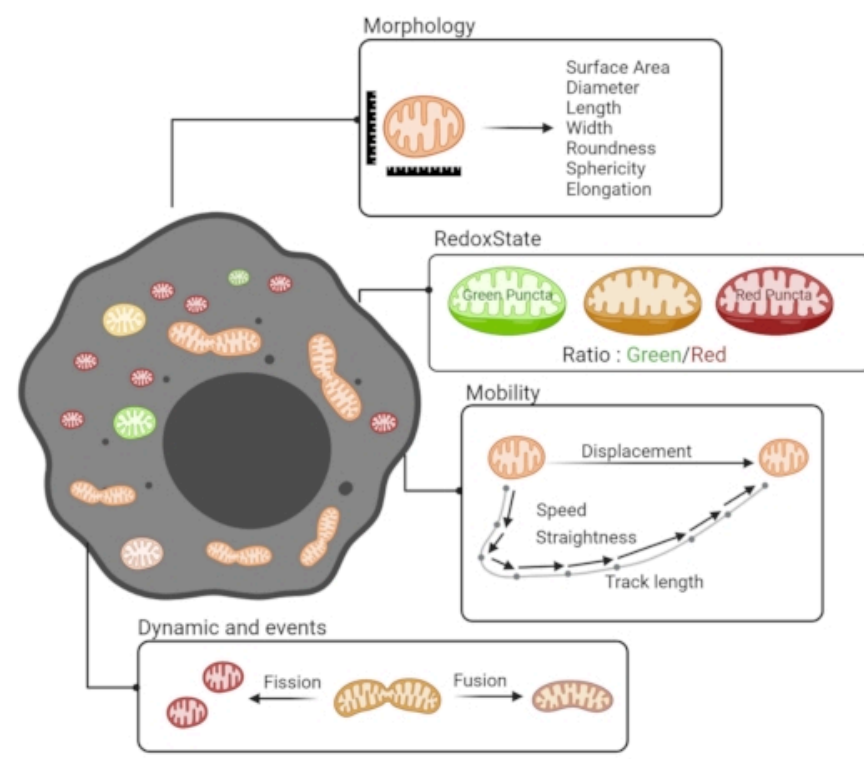

Figure 1: Astrocyte culture expressing LV-G1-MitoTimer biosensor. (A) Confocal photographs of astrocytes expressing LV-G1-MitoTimer. (B) Selection of confocal photographs of reduced (green) balanced (orange) and oxidized (red) mitochondria with different levels of fragmentation. (C) Summary diagram of the different criteria available for analysis in an astrocyte expressing LV-G1-MitoTimer. Scale bar: (A) Upper panel: $50 \mu \mathrm{m}$, lower panel: $10 \mu \mathrm{m}$, (B) $1 \mu \mathrm{m}$. Please click here to view a larger version of this figure. 

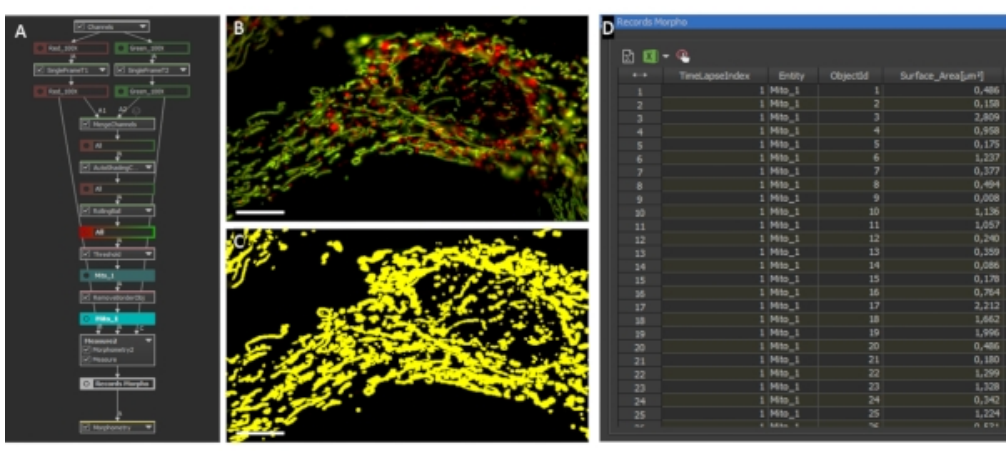

Figure 2: Mitochondrial morphology and ratio analysis. (A) GA3 script overview for the analysis of individual mitochondrial morphology and ratio. (B) Initial photographs of an astrocyte expressing LV-G1-MitoTimer analyzed with the GA3 script. (C) Example of binary masks generated for the mitochondrial system of astrocytes. Scale bar: $10 \mu \mathrm{m}$ (B-C). Please click here to view a larger version of this figure. 

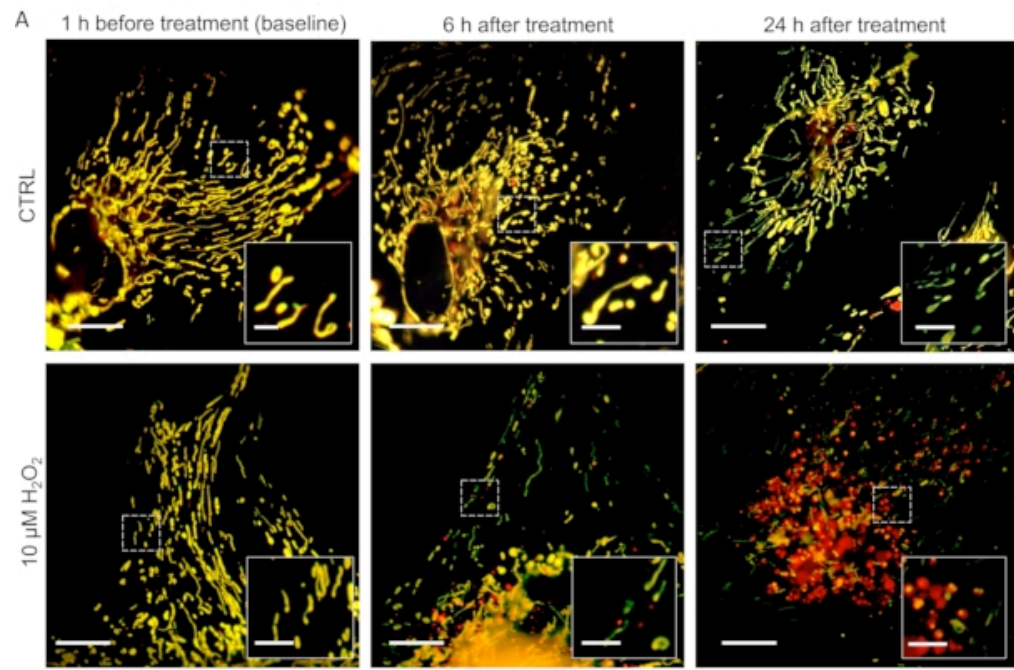

B

Morphology

-. Baseline

$10 \mu \mathrm{M} \mathrm{H}_{2} \mathrm{O}_{2}$

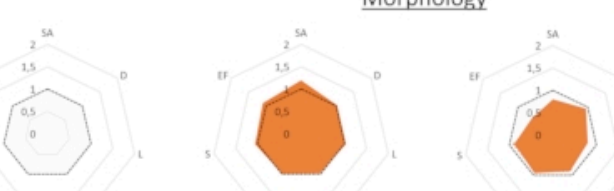

$\underline{\text { Redox State }}$

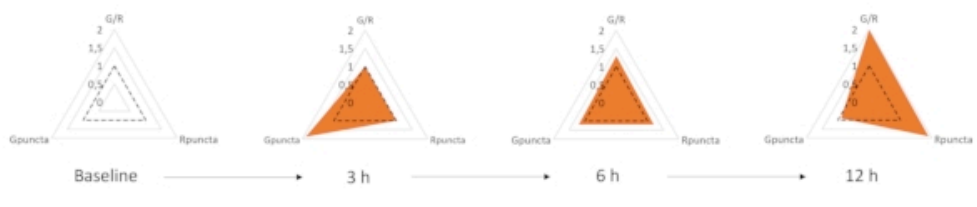

Mobility

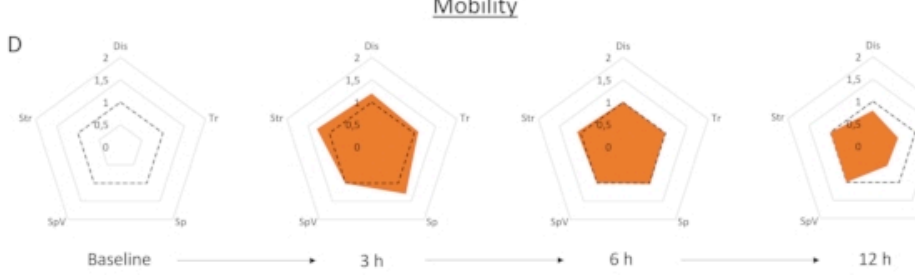

Figure 3: The effects of $\mathrm{H}_{2} \mathrm{O}_{2}$ on the mitochondrial system of astrocytes. (A) Photographs of astrocytes expressing
LV-G1-MitoTimer $1 \mathrm{~h}$ before and $6 \mathrm{~h}, 24 \mathrm{~h}$ after treatment with PBS (CTRL) and $10 \mu \mathrm{M}$ of $\mathrm{H}_{2} \mathrm{O}_{2}$.
(B) Radar charts of

mitochondrial morphology, (C) redox state and turnover, and (D) mobility criteria evaluated on astrocytes during baseline and $3 \mathrm{~h}, 6 \mathrm{~h}$, and $12 \mathrm{~h}$ after $\mathrm{H}_{2} \mathrm{O}_{2}$ treatment. SA: Surface area; D: Diameter; L: Length; W: Width; R: Roundness; S: Sphericity; EF: Elongation factor (=L/W); G/R: Individual red/green ratio; Gpuncta: Percentage of green puncta mitochondria; Rpuncta: Percentage of red puncta mitochondria; Dis: Displacement; Tr: Track Length; Sp and SpV: Speed and speed variance; Str: Straightness. Scale bar: $20 \mu \mathrm{m}(\mathrm{A})$ and $2.5 \mu \mathrm{m}$ (inset). Please click here to view a larger version of this figure. 

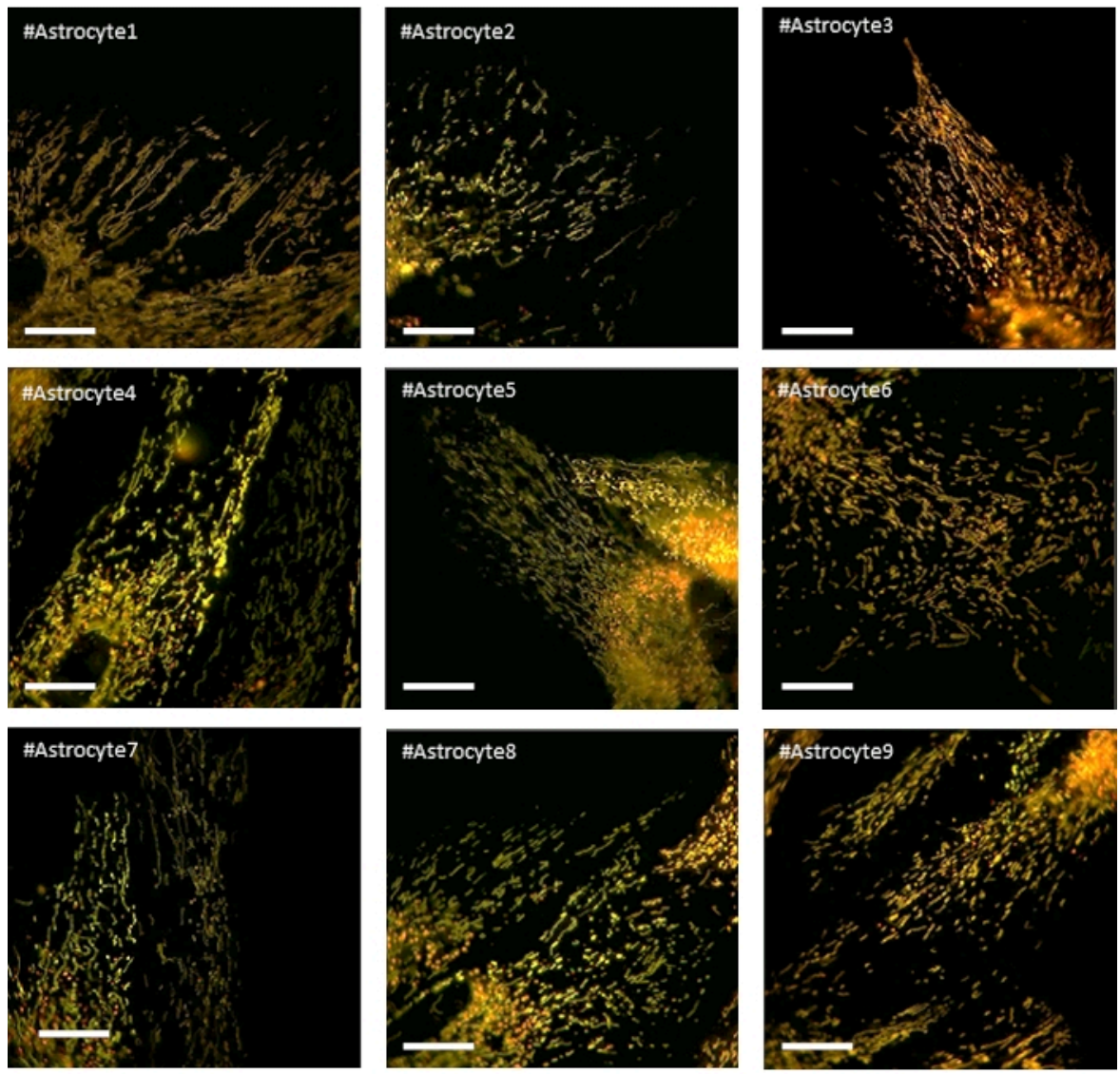

Figure 4: Photographs of astrocytes expressing LV-G1-MitoTimer and showing a homogeneous and balanced mitochondrial network during baseline. Scale bar: $20 \mu \mathrm{m}$ Please click here to view a larger version of this figure.

Video 1: Effect of $\mathrm{H}_{2} \mathrm{O}_{2}$ treatment on the mitochondrial system of cultured astrocytes. Astrocytic mitochondria before $\mathrm{H}_{2} \mathrm{O}_{2}$ treatment (baseline), as well as $6 \mathrm{~h}$ and $24 \mathrm{~h}$ after $\mathrm{H}_{2} \mathrm{O}_{2}$ treatment compared to non-treated control cell. Please click here to download this File.

\begin{tabular}{|c|c|c|}
\hline Morphology criteria & Range & Remarks \\
\hline Surface Area (SA) & $0.5-4 \mu \mathrm{m}^{2}$ & \multirow{6}{*}{$\begin{array}{l}\text { These criteria inform on the fragmented-elongated features } \\
\text { of mitochondria. They generally evolve in the same direction. } \\
\text { Fragmented mitochondria will have decreased surface area, } \\
\text { diameter, length, and elongation factor while roundness, } \\
\text { sphericity, and width may be unchanged or increased. }\end{array}$} \\
\hline Diameter (D) & $0.5-1.5 \mu \mathrm{m}$ & \\
\hline Length (L) & $0.5-5 \mu \mathrm{m}$ & \\
\hline Width (W) & $0.5-2 \mu \mathrm{m}$ & \\
\hline Roundness (R) & $0-1$ & \\
\hline Sphericity (S) & $0-1$ & \\
\hline
\end{tabular}


Elongation factor

$(\mathrm{EF}=\mathrm{L} / \mathrm{W})$

Table 1: Summary of selected parameters for mitochondrial morphology.

\begin{tabular}{|c|c|c|}
\hline Redox State criteria & Range & Remarks \\
\hline Individual ratio (G/R) & $0-10$ & \multirow{3}{*}{$\begin{array}{l}\text { The ratio indicates the result of the redox state. It informs about the } \\
\text { general state and age of the mitochondria in the cell. It is essential to } \\
\text { consider that this ratio is the balance of biogenesis and degradation } \\
\text { of mitochondria and the fission/fusion of oxidized mitochondria with } \\
\text { reduced mitochondria. Therefore the evaluation of the number of } \\
\text { green and red puncta can powerfully help interpret the results. Green } \\
\text { puncta mitochondria are determined when the intensity of green is } 10 \\
\text { times that of red. Red puncta mitochondria are determined when the } \\
\text { intensity of red is } 10 \text { times greater than that of green. The redox state } \\
\text { of an astrocyte is the average of all the mitochondria ratios of that cell. }\end{array}$} \\
\hline $\begin{array}{c}\text { Percentage of } \\
\text { green puncta } \\
\left.\text { mitochondria ( } G^{\text {puncta }}\right)\end{array}$ & $0 \%-100 \%$ & \\
\hline $\begin{array}{c}\text { Percentage } \\
\text { of red puncta } \\
\left.\text { mitochondria ( } R^{\text {puncta }}\right)\end{array}$ & $0 \%-100 \%$ & \\
\hline
\end{tabular}

Table 2: Summary of selected parameters for mitochondrial redox state.

\begin{tabular}{|c|c|c|}
\hline Mobility criteria & Range & Remarks \\
\hline Displacement (Dis) & $0-10 \mu \mathrm{m}$ & \multirow{5}{*}{$\begin{array}{l}\text { Together these features inform the general motility dynamics of the } \\
\text { network. Stationary mitochondria display short displacement \& track } \\
\text { length with a low speed. On the other hand, oscillatory particles can be } \\
\text { differentiated with a difference between the track length and displacement } \\
\text { (resulting in low straightness) and an increased speed compared to static. }\end{array}$} \\
\hline Track Length (Tr) & $0-10 \mu \mathrm{m}$ & \\
\hline $\begin{array}{c}\text { Speed and speed } \\
\text { variance (Sp and SpV) }\end{array}$ & $0-1.5 \mu \mathrm{m} / \mathrm{s} \pm 0.2 \mu \mathrm{m} / \mathrm{s}$ & \\
\hline Straightness & \multirow[t]{2}{*}{$0-1$} & \\
\hline $\begin{array}{c}(\text { Str }=\text { displacement } / \\
\text { track length })\end{array}$ & & \\
\hline
\end{tabular}

Table 3: Summary of selected parameters for mitochondrial mobility. 


\section{Supplemental Coding File 1: GA3 script file for analysis} of individual mitochondrial morphology. Please click here to download this File.

\section{Discussion}

Here, a novel method to longitudinally follow the dynamics and turnover of the mitochondrial system in a cultured astrocyte is proposed. Unlike a time-lapse approach on a fixed group of cells or one individual cell at a time (most often used in the literature $)^{24,25}$, researchers can follow the evolution of the mitochondrial system during several days on the same individual cells. In contrast to single well live imaging where high levels of light exposure are required, and the selection of many individual cells is less feasible, the proposed method takes advantage of this microscope's ability to image several different cells in different areas of a well and to come back to those same cells at various time points to re-image them. Thanks to normalization to a baseline carried out for each measured criterion on each cell of interest, it takes the mitochondrial system's complexity into account and investigates the effect of treatment on each cell relative to its own baseline image. The microscope's ability to autonomously carry out this type of imaging on up to 16 wells at a time (imaging 5 cells per well) allows for the heterogeneity of the mitochondrial system to be properly taken into account during analysis without the experimental variability that comes with imaging various conditions on different days.

The quality of the cultures, the levels of viral infections expressing the LV-G1-MitoTimer biosensor, the type of microscope and objectives, and the selection of suitable cells are critical variables that must remain as consistent as possible in this protocol. The cell densities, the type of vector, and the viral titers can be adapted according to the question. Although previous work shows that LVG1-MitoTimer expression has no deleterious consequences for mitochondrial function and dynamics $21,22,26,27$, it is essential to verify that the concentration is not toxic for the cells (for example, checking the total number of cells in control well). As a single focal plane is used, astrocytes should be: (1) as flat as possible, (2) isolated from other labeled cells (to simplify the analysis in case of displacement in the dish), and (3) possessing high fluorescence levels. As cells in culture can be highly variable in morphology, the mitochondrial system can be highly heterogeneous. In this context, analyzing ROls (and not the whole cell) compensates for some problematic regions, such as the perinuclear regions, and decreases variability. It is essential to do the baseline on relatively similar cells and sample as many cells as possible. Consequently, high content acquisition and analysis microscopes are ideally suited. During this longitudinal monitoring, it is also important not to overexpose the cells to light to avoid biosensor bleaching.

This imaging method is not without its complexities, and throughout the protocol, several notes are included, which take into account troubleshooting done during previous tests with the microscope. For example, the choice of plate coating used depends on the intended assay, but recommendations for the most suitable choices for astrocyte primary cultures have been included. Additionally, image acquisition should be performed on at least 5 cells per condition due to intercellular variability. More specifically, some cells selected at baseline imaging will die, some will move out of the frame of the assigned image acquisition area, and some will change their morphology, making the mitochondria very difficult to individualize in analysis. Imaging many cells from the beginning increase the likelihood of a large enough sample size of cells to analyze at the end of the experiment. 
In addition to the more complex aspects of this imaging technique, there are some outright limitations as far as who can benefit from this type of imaging and analysis. In order to take full advantage of the automatization of image acquisition, the microscope used must have an autofocus system that can handle the speed of time intervals between images (i.e., every $3 \mathrm{~s}$ in this protocol) and can consistently focus on the cell in question before each image is taken. Additionally, without the JOBS software, which automates the entire image acquisition process, this method becomes arduous and potentially impossible depending on the number of cells being imaged as it would require manually finding and imaging each cell again at the appropriate time point. Finally, this imaging method is not immune to the issue of photobleaching. For this reason, as with any long-term acquisition method, it is important to choose fluorescent markers that are less susceptible to photobleaching and to tailor image acquisition to avoid this issue as much as possible.

This technique differs from others currently used in a crucial way. Unlike other time-lapse studies, this technique does not require imaging on the same position in the well the entire time, nor does it require manual movement of the plate to image other areas. This allows researchers the ability to image many cells in many conditions in one $24 \mathrm{~h}$ timeframe. Consequently, the ability to carry out this imaging and analysis on many cells in each well gives the same population information one would obtain from broadly studying a large group of cells while additionally providing specific measures from each cell imaged. While some specificities to this method may not apply to other image acquisition methods (outlined above), the benefits outweigh the complications with the type of analysis possible after acquisition. This technique allows researchers to see the exact ramifications of various treatments on the mitochondrial system, and consequently, on the cultured astrocytes.

Additionally, this method is highly customizable to many different scientific questions regarding mitochondrial behavior and roles in specific contexts. Here the outlined protocol deals specifically with cultured astrocytes. However, many other cell types can be used, and the treatments that can be tested are limited only by the questions being investigated. This type of imaging has the potential to advance the collective knowledge and understanding of mitochondrial behavior, the underlying mechanisms that lead to mitochondrial dysfunction, and the effects of many pathologies on the innate dynamics present in different types of cells.

\section{Disclosures}

The authors declare no competing interests.

\section{Acknowledgments}

This study was supported by a Synapsis Foundation fellowship awarded to K.R. and the Lausanne University Hospital (CHUV). The authors thank Nikon for their help, in particular J. Gannevat.

\section{References}

1. Ballabh, P., Braun, A., Nedergaard, M. The bloodbrain barrier: An overview: Structure, regulation, and clinical implications. Neurobiology of Disease. 16 (1), $1-13(2004)$.

2. Allen, N.J., Eroglu, C. Cell biology of astrocyte-synapse interactions. Neuron. 96 (3), 697-708 (2017).

3. Bernardinelli, Y., Muller, D., Nikonenko, I. Astrocytesynapse structural plasticity. Neural Plasticity. 2014, 232105 (2014). 
4. Benarroch, E. E. Neuron-astrocyte interactions: Partnership for normal function and disease in the central nervous system. Mayo Clinic Proceedings. 80 (10), 1326-1338 (2005).

5. MacVicar, B. A., Choi, H. B. Astrocytes provide metabolic support for neuronal synaptic function in response to extracellular K+. Neurochemical Research. 42 (9), 2588-2594 (2017).

6. Song, H., Stevens, C. F., Gage, F. H. Astroglia induce neurogenesis from adult neural stem cells. Nature. 417 (6884), 39-44 (2002).

7. Christopherson, K. S. et al. Thrombospondins are astrocyte-secreted proteins that promote CNS synaptogenesis. Cell. 120 (3), 421-433 (2005).

8. Volterra, A., Liaudet, N., Savtchouk, I. Astrocyte Ca2+ signalling: An unexpected complexity. Nature Reviews Neuroscience. 15 (5), 327-335 (2014).

9. Huntington, T. E., Srinivasan, R. Astrocytic mitochondria in adult mouse brain slices show spontaneous calcium influx events with unique properties. Cell Calcium. 96, 102383 (2021).

10. Kofuji, P., Newman, E. A. Potassium buffering in the central nervous system. Neuroscience. 129 (4), 1045-1056 (2004).

11. Ridet, J. L., Malhotra, S. K., Privat, A., Gage, F. H. Reactive astrocytes: Cellular and molecular cues to biological function. Trends in Neurosciences. 20 (12), 570-577(1997).

12. Liddelow, S. A., Barres, B. A. Reactive astrocytes: Production, function, and therapeutic potential. Immunity. 46 (6), 957-967 (2017).
13. Young, A., Gill, R., Mailloux, R.J. Protein Sglutathionylation: The linchpin for the transmission of regulatory information on redox buffering capacity in mitochondria. Chemico-Biological Interactions. 299, 151-162 (2019).

14. Lackner, L. L. Shaping the dynamic mitochondrial network. BMC Biology. 12, 35 (2014).

15. Willems, P. H. G. M., Rossignol, R., Dieteren, C. E. J., Murphy, M. P., Koopman, W. J. H. Redox homeostasis and mitochondrial dynamics. Cell Metabolism. 22 (2), 207-18 (2015).

16. de Michele, R., Carimi, F., Frommer, W. B. Mitochondrial biosensors. International Journal of Biochemistry and Cell Biology. 48, 39-44 (2014).

17. Terskikh, A. et al. "Fluorescent timer": Protein that changes color with time. Science. 290 (5496), 1585-1588 (2000).

18. Rizzuto, R. et al. A gene specifying subunit VIII of human cytochrome c oxidase is localized to chromosome 11 and is expressed in both muscle and non-muscle tissues. Journal of Biological Chemistry. 264 (18), 10595-10600 (1989).

19. Rizzuto, R., Brini, M., Pizzo, P., Murgia, M., Pozzan, T. Chimeric green fluorescent protein as a tool for visualizing subcellular organelles in living cells. Current Biology. 5 (6), 635-642 (1995).

20. Ferree, A. W. et al. MitoTimer probe reveals the impact of autophagy, fusion, and motility on subcellular distribution of young and old mitochondrial protein and on relative mitochondrial protein age. Autophagy. 9 (11), 1887-1896 (2013). 
21. Hernandez, G. et al. MitoTimer: A novel tool for monitoring mitochondrial turnover. Autophagy. 9 (11), 1852-1861 (2013).

22. Richetin, K. et al. Tau accumulation in astrocytes of the dentate gyrus induces neuronal dysfunction and memory deficits in Alzheimer's disease. Nature Neuroscience. 23 (12), 1567-1579 (2020).

23. Merienne, N. et al. Gene transfer engineering for astrocyte-specific silencing in the CNS. Gene Therapy. 22 (10), 830-839 (2015).

24. Sison, M. et al. 3D Time-lapse imaging and quantification of mitochondrial dynamics. Scientific Reports. 7, 43275 (2017).

25. Miyazono, Y., Hirashima, S., Ishihara, N., Kusukawa, J., Nakamura, K. I., Ohta, K. Uncoupled mitochondria quickly shorten along their long axis to form indented spheroids, instead of rings, in a fission-independent manner. Scientific Reports. 8 (1), 350 (2018).

26. Trudeau, K. M., Gottlieb, R. A., Shirihai, O. S. Measurement of mitochondrial turnover and life cycle using MitoTimer. Methods in Enzymology. 547, 21-38 (2014).

27. Stotland, A., Gottlieb, R.A. a-MHC MitoTimer mouse: In vivo mitochondrial turnover model reveals remarkable mitochondrial heterogeneity in the heart. Journal of Molecular and Cellular Cardiology. 90, 53-58 (2016). 